\title{
Meta
}

Journal des traducteurs

Translators' Journal

\section{Le rôle linguistique du français et de l'anglais dans l'expression des concepts techniques et semi-techniques dans les langues africaines}

\section{Kweku A. Sampson}

Volume 40, numéro 1, mars 1995

URI : https://id.erudit.org/iderudit/004024ar

DOI : https://doi.org/10.7202/004024ar

Aller au sommaire du numéro

Éditeur(s)

Les Presses de l'Université de Montréal

ISSN

0026-0452 (imprimé)

1492-1421 (numérique)

Découvrir la revue

Citer cet article

Sampson, K. A. (1995). Le rôle linguistique du français et de l'anglais dans l'expression des concepts techniques et semi-techniques dans les langues africaines. Meta, 40(1), 73-80. https://doi.org/10.7202/004024ar 


\section{LE RÔLE LINGUISTIQUE DU FRANÇAIS ET DE L'ANGLAIS DANS L'EXPRESŞION DES CONCEPTS TECHNIQUES ET SEMI-TECHNIQUES DANS LES LANGUES AFRICAINES}

KWEKU A. SAMPSON

Université du (Ghina. Legon. Acrra, Ghäna

Les langues africaines sont, de toute évidence, appelées à relever le défi de l'expression technique dans un monde dominé par la science et la technologie. Nous nous proposons ici de nous pencher sur les principaux outils linguistiques utilisés par un grand nombre de langues africaines pour la désignation des référents techniques ou semitechniques. Il s'agit surtout de la translittération, de la traduction, ainsi que des formules paraphrastiques et périphrastiques traduites. Notre propos, dans un premier temps, consistera à définir au plan linguistique ces trois derniers procédés avant de passer, dans un deuxième temps, aux illustrations. êt d'évoxquer brièvement d'autres possibilités de création lexicale dans le domaine des registres plus ou moins spécialisés.

La translittération et la traduction. quoique non identiques, partagent un trait linguistique fondamental en ce sens qu' elles impliquent une activité de transfert qui va d'une langue source (LS - en l'occurrence le français ou l'anglais selon le cas) vers une langue cible (LC - la langue africaine en question). La différence réside dans le fait qu'en ce qui concerne la translittération, le lexème français ou anglais est adapté à la structure phonéticographique de la langue source qui l'accueille. C'est ainsi que Jean Dubois et al. (1973) signalent que la translittération intervient "quand on veut représenter dans un système d'ecriture une suite de mots d'une langue utilisant généralement un autre système d'écriture».

On rencontre communément dans les langues d'Afrique de telles adaptations lexicales que certains auteurs, dont Mateene (1979: 51), considèrent comme étant une source d'enrichissement. Ainsi, dans Lexique's spécialisés peruls (MAPE 198.3) les linguistes du projet MAPE (Promotion des langues mandingues et peuls) consignent des cas relevant de la translittération et impliquant une tentative d'adaptation des structures phonologiques et orthographiques du français a celles du peul. Au niveau des consonnes, par exemple. l'absence de la constrictive labio-dentale sonore /V/ en peul (Arnoll 1970: 41 ; De Guiraudon 1894: 4: Taylor 1953: 1) fait que les mots français suivants donnent lieu aux translittérations correspondantes en peul :

Witamine (vitamine): neiriri (vitre): n'erre (verre) (MAPE 1983: 37)

De même, la constrictive dentale sonore /Z/ étant généralement manquante en peul (Taylor 1953: 2), ce phonème a tendance à se désonoriser pour devenir sourd en peul comme dans gaas (gar): gacssiual (gas-oil) ou à céder la place à un substitut consonantique : majut (mazout) (Léxiques spécicalisés peuls: 126, 130). Au niveau des voyelles, certains phonèmes vocaliques trançais qui n'existent pas en peul sont remplacés : c'est le cas des voyelles /at et /y/du français moteer (moteur): temperatiir (température) (MAPE 1983: 130, 135). 
De même. le système orthographique du peul, tout comme celui d'autres parlers africains, s'adapte pour permettre l'intégration de certains mots d'emprunt. Les linguistes du projet MAPE font état d'une tendance fréquente concernant les mots français qui comportent le c orthographique. Celui-ci. dans les translittérations en peul, devient $k$ dans l'écriture: karbomik (carbonique); kirivon (crayon): kibbu (cube): kave' (cahier): mikroskope' (microscope): me’kanth (mécanique): c'kuru (écrou); hak (bac) (MAPE 1983).

Des mots issus du processus de translitteration foisonnent également en akan (langue la plus répandue au Ghāna) et nous en discutons largement ailleurs (Sampson 1988). Retenons qu'en général. les modalités linguistiques de la translittération dépéndent surtout des structures phonologiques et orthographiques de la langue cible, dans la mesure où celle-ci cherche à intégrer des éléments lexicaux venant d'une autre langue. Én règle générale, le nombre de mots d'emprunt adaptés à une langue cible quelconque peut être déterminé par la volonté des usagers el des décideurs politiques à recourir à ce processus d'extension du répertoire lexicologique de la langue en question. En effet, il est remarquable qu 'en somali. la translittération n’intervient pas beaucoup dans la recherche et la création d'un vocabulaire relatif aux référents dits umodernes». ("est parce que la politique linguistique du somali privilégie des expressions et des mots propres à la langue du pays plutôt que des emprunts (Andrzejewski 1971). Ajoutons également que le Cameroun, pays officiellement bilingue, représente dans certains cals un environnement linguistique où l'on peut difficilement déterminer de façon claire et nette si cest le français ou l'anglais qui a fourni telle ou telle translitteration. Mbassi-Mbanga (1979: 59-60) cite des translitterations en duala qui illustrent ce probleme: jeografi (de géographie ou de geography ?): frensi (de français ou de fronch ?").

La traduction des termes français ou anglais vers les langues africaines est un autre outil important dans le domaine de l'expression lechnique et semi-technique en Afrique. À titre d'illustration. nous examinerons quelquess exemples tirés de la métalangue utilisée dans les grammaires akans rédigées en cette même langue. Bartels et Annobil (1960: 1-2), dans leur grammaire du fanti (dialecte de l'akan), utilisent une terminologie métalinguistique dont la majeure partice est issue de traduction des termes anglais. ces termes sources étant indiqués par les auteurs cux-mêmes à côté des versions fantis. ("est ainsi qu a partir de l'anglais, les notions adverbiales de manière. de lieu, de concession et de but sont respectivement rendues en fanti par nyebea (la manière ou la façon de faire) : béd (lieu): ngyétomu (le fait de concéder ou d'admettre): botae (finalité ou but). Akrofi (1965: 26. 48) qui a également écrit une grammaire en twi sur ce dernier dialecté akan, fournit luimêtme les mots sources anglais qui. par le truchement de lat traduction. ont donné licu aux versions akans suivantes:

adjectif : nkyerekveremu (ce qui explique ou qualifie):

- conjonction : nkabombe (ce qui permet d'unir).

À l'instar d'Akrofi. Bartels et Annobil ( 1960 : 2 ) proposent, toujours en traduisant à partir de l'anglais, less lexèmes métalinguistiquess suivants:

- nsienvim (ce qui est placé avant); et

- nsiekvir (ce qui est placé aprés).

ces deux derniers élant pour "préfixe" et "suffixe" respectivement. Anoh-Kumi (1979), dans son travail en akan sur la phonologie de cette même langue, se base également sur la

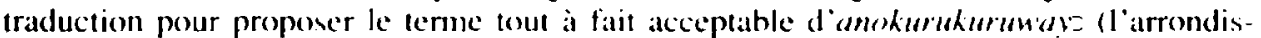
sement des levres) pour «labialisation». Fin somali. Andracjewski (1971: 268-27()) signale des procédés de designation relevant de la traduction. lese ondes courtes, par exemple. sont appelées hirarka gauggasuban, ce qui, selon l'auteur en question, signifie ades ondes 
qui sont courtes». Dans le même ordre d'idées, wasaarades w'arfaafinta désignant le ministère de l'Information émane d'une traduction et signifie "ministère qui répand des informations».

Après la traduction. passons à l'utilisation dans les langues africaines de formules paraphrastiques et périphrastiques hasées sur des lexèmes ou syntagmes provenant de l'anglais ou du français pour la dénomination technique et semi-technique. On relève chez. J. Dubois el al. (1973) une double définition linguistique de la paraphrase: d'une part une conception un peu restreinte, selon laquelle une séquence, A, est jugée comme étant une paraphrase d'une autre séquence, $B$, s'il y a identité ou coïncidence sémantique au niveau du contenu de $\mathrm{A}$ et $\mathrm{B}$. La deuxième caractérisation est plus étendue et plus répandue : «on appelle paraphrase le développement explicatif d'une unité ou d'un texte». Dans ce cas. B, en tant que paraphrase, se veut relativement plus transparent, simple accessible sur le plan sémantique par rapport à $\mathrm{A}$. ce qui nécessite parfois, au niveau de B. une décomposition ou une explication sémantique du contenu de A. Dans le cadre des discussions qui suivent. il sera question non seulement de paraphrases, mais aussi de périphrases, étant entendu que sur le plan linguistique. les deux relèvent du phénomène de synonymic. la périphrase étant souvent synonyme d'un lexème (ex. "femelle d'un cheval» pour "jument") alors que la paraphrase lend à être synonymique par rapport à une séquence plus longue. Retenons que la quasi-totalité des illustrations paraphrastiques et périphrastiques qui suivent sont tirées de textes où les auteurs eux-mêmes ont pris soin de signaler entre parenthèses les sources anglaises ou françaises ayant inspiré leurs versions africaines. À des fins de comparaison, nous reprenons à notre tour, toujours entre parenthèses, les termes anglais ou français de départ qui selon nos textes de référence constituent la source des exemples cités.

Deux problèmes théoriques méritent d'être examinés avant d'évoquer des cas concrets relatifs aux formules paraphrastiques et périphrastiques. Tout d'abord, comme il s'agit de séquences traduites vers l'akan, le somali, ete., la question se pose de savoir si le processus de transformation en paraphrase ou en périphrase, selon le cas, précède ou suit la traduction. À cet égard, deux possibilités théoriques sont envisageables et peuvent s’illustrer comme suit avec l'anglais et l'akan.

(a) La version anglaise est transformée en paraphrase ou en périphrase (selon le cas) dès la langue source, el ce, avant d'être traduite vers la langue africaine en question:

ANG $/ F R(N o n P) \rightarrow A N G / F R(P) \rightarrow A F(t)$

Version angl. ou fr. non paraphrasće devient paraphrasée et donne lieu après traduction à la version africaine traduite ou $\mathrm{AF}(\mathrm{tr})$.

(b) La deuxième possibilité théorique serait d'avoir une version anglaise ou française non paraphrasée qui donne directement lieu à une version traduite en langue africaine. celle-ci étant par la suite paraphrasée :

$$
A N G / F R(N o n P) \rightarrow A F(t r) \rightarrow A F(P)
$$

En un mot, il n'est pas facile de discerner $s^{\prime} i l$ s'agit (a) de formules paraphrastiques (anglaises ou françaises) traduites vers les langues africaines ou de (b) traductions (en langues africaines) qui deviennent ensuite des paraphrases dans ces mêmes langues.

La deuxième difficulté théorique est relative à la distance sémantique éventuelle entre la version anglaise ou française de dépan ( $A N G / F R(N o n)$ )) et la version akan. En efiet, la version en langue africaine ne saurait être acceptable si l'écart qui la sépare de la version originale esı de nature à fausser ou à falsifier le contenu sémantique de la version en langue africaine. C'est dire qu'il y a lieu de se pencher également sur la proximité ou l'écart sémantique de la version africaine par rapport à son homologue français ou 
anglais. Pour ce faire. les paraphrases et périphrases que nous présenterons seront catégorisées selon le critère sémantique de proximité référenticlle. c'est-à-dire selon le degré d'identité. de coinncidence entre les référents dans la version en langue source (anglais ou français) et ceux de la paraphrase en langue africaine (akan, par exemple). La distance référentielle peut être déterminéc grâce à une analyse lexicographique du contenu sémantique des deux versions (anglaise et akan. èl l'oceurrence).

(a) La première catégorie marquéc (S (coincidence sémantique) réunit des paraphrases et périphrases en langue africaine dont les référents peuvent être considérés comme présentant une identité ou coïncidence totale ou presque par rapport aux référents des versions anglaises correspondantes.

(b) Le deuxième groupe. marque PS (proximité sémantique) comporte des cas où les référents en anglais el en akan sont assez proches mais plus distants que ceux de la catégorie A. Nous tâcherons, dans la mesure du possible, de montrer en quoi réside l'écart sémantique: celui-ci peut généralement provenir d'erreurs liées soit à la traduction, soit au processus d’élaboration paraphrastique (ou périphrastique).

Passons maintenant aux exemples:

Catégorie A: CS (Coincidence ou identité sémantique au niveau des référents dans la version originale el la paraphrase). Jusse à côté de l'akan, nous indiquons entre parenthèses le terme anglais ou français que les atteurs eux-mêmes fournissent comme source de lat paraphrase ou périphrase en langue africaine. Ce terme source est suivi de la mention "L.S" (langue source). Pour rendre accessible le contenu de l'expression akan, nous la traduisons nous-même.

I. Akan : Avarsabos kakrobat lolinic . I.S : ang.) trad. fras. Petit endroit ois l'on guérit les maladies. (clinique) trad. ang. : Small place nhere ailme'ms are he'sled. Exie: Wonsom (19)6o: 3).

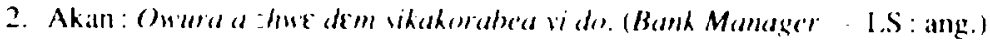
trad. frat : Le responsiable de l'établissement où l'on dépose de l'argent. (directeur de banque) trad. ang. : Persom in sharge of the place where money is hept. lexte: Wonsom (1987:5).

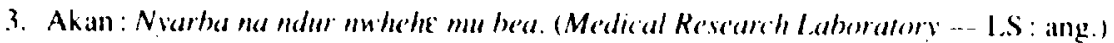
trad. fra. : In endroit où l'on mente des recherches sur les maladies et les medicaments. (laboratoire de recherche medicale) trad. ang. : A place where research is comducted imto discesses and drugs. lexte: Wonsom (1087: 10).

4. Akan : Niquakan. (Populasiom ce'n.was - I.S : ang.) trad. fra. : Dénombrement des populations. (recensement) trad. ang. : comming of porple' texte: Census Secretarial (1960)

5. Somali : Qiida ilmada he'ena. (le'sr gas - I.S : ang.) trad. fra. : Fumec qui provorque des larmes. (gatz lacrymogene) trad. ang. : Smoke that cosesse's tears. Texte : Andraejewski (1971: $27(1)$.

6. Somali : Midabkalasoor (racial discrimination --. 1.S : ang.) trad. fra. : Separation sur la base de la couleur. (discrimination raciale) rad. ang : Separation on the hasis of colosur. texte: Andrzejewski (197): 268$)$. 
7. Ewe : Levi alo nugbaghe set si wogayona ha he dolékui (microbe - LS français)

trad. fra. : Un petit objet vivant ou bien le plus petit objet vivant qu on appelle aussi agent causateur de maladie.

trad. ang. : A small living object or the smallest living object also called a disease-causing agent.

texte: Adzomada (1971: 61).

8. Akan : Adwenkvere ambatow' (referendum - LS : ang.)

trad. fra. : Vote permettant d'exprimer une opinion.

trad. ang. : Voting by which opinion is expressed. texte : ministère de l'Information (1965).

Catégorie B : Proximité au niveau des référents dans la version d'origine (exemple anglais) et la version paraphrastique. Il s'agit là d'un écart relativement réduit entre les référents en LS et LC. Cela peut émaner de trois facteurs principaux que nous évoquons avec des illustrations.

Catégorie B1: On observe parfois dans la paraphrase (en LC) qu'il existe une restriction sémantique ou une réduction de la gamme de référents que comporte la version originale.

1. Akan: Akontaabude (numerals - LS : ang.)

trad. fra. : Ce qui sen à faire des calculs. (numéraux)

trad. ang. : That which is used for callulation.

texte: Akrofi (1965: 34).

La périphrase akan conçoit les numéraux comme permettant seulement d'effectuer des calculs, laquelle conception est quelque peu restrictive.

2. Akan : Efir a sma nduadzew'a n'on'. (solar dryer - LS : ang.) Irad. fra.: Machine à faire sécher les aliments.

trad. ang. : Machine which drys food items

texte: Wonsom (1989: 34).

À la différence de la version anglaise qui en est la source, la paraphrase akan limite les matières humides susceptibles d'être séchées par ce dispositif aux seules denrées alimentaires, ce qui constitue une restriction de la gamme référentielle.

3. Akan: (jya dumfo. vire men - L.S : ang.)

trad. fra. : Ceux qui combattent les incendies. (sapeurs-pompiers)

trad. ang.: Those who put out fire

texte: Wonsom (1986: 5 ).

La restriction référentielle provient du fait qu en réalité les sapeurs-pompiers combattent non seulement le feu. mais aussi d'autres sinistres.

4. Akan : Ghina amamfo mpanyimfo akyerew na akenkan do mo. (Cihina National Council for Adult Education - LS: ang.)

Irad. fra.: L'organe national du Ghāna chargé d'enseigner aux adultes l'écriture et la lecture. (Conseil National du (ihāna chargé de l'éducation des adultes)

trad. ang. : National body of (Fhina responsible for teac hing adults reading and writing.

texte: Wonsom (1987: 3).

L'éducation des adultes, en tant que discipline, est réduite ici à l'enseignement de la lecture el de l'écriture. Mais en anglais comme en français. cette branche d'activités recouvre un domaine plus vaste.

Catégorie B2: Adjonction ou extension sémantique; dans ce cas, la paraphrase, qui n est pas pour autant inacceptable. s'avère sémantiquement trop élastique et pas suffisamment précise.

5. Somali : Hoyga madaddaalada. (Nasional theatre)

trad. fra. : Maison destinée aux divertissements. (Thểtre national) 
trad. ang. : Housc for entertainme'nt.

texte: Andraejewski (1971:268).

Le Théâtre national est génératement le lieu des spectacles liés à l'art dramatique. Cependant, la désignation en langue somali recouvre de façon élastique d'autres types de divertissements ne relevant pas forcement de la dramilturgie.

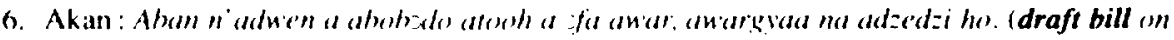
marriage. divorec and inherifance $\mathrm{ISS}$ : ang.)

trad. Fra.: (ce que le gouvernement prévoit en matière de mariage, de devorce et de succession. (projet de loi sur le marage. ke divorce et la succession)

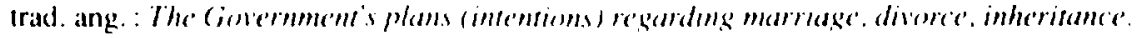
kexke: Burcau of (ihina Languatgen (196) 1).

La paraphrase akan qui est cencéce rendre la notion de projet de loj est asses diluée el se résume simplement à ce gu une entité (le gouvernement) se propose de faire ou prévoit. L.al formulation ataln est donc sous-lendue par un referent asses vague qui. par rapport à loriginal. est dépourvu de valeur jurictique et de précoston référentielle.

Catégorie B3: Chevatuchement au niveau de la paraphrase (ou périphrase) entre les référents visés dans la version originale el d'autres éléments référentiels connexes.

Les ouvrages de grammaire akan deja cites foumissent quelques exemples de ce problème de différenciation el d'individualisation référentielle. En effet. Akrofi (1965: 11) et Anoh-Kumi (1971) emploient les lermes akans suivant pour "consonne" et "voyelle» respectivement :

7. Akan : Enhe moviguri. (rencel-I.S: ang.)

trad. frat. Son dam la volix. (voyelle)

trad. ang. : Sound in the verter

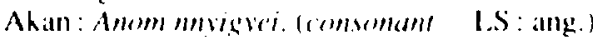

trad. Pra. : Son dam la bouche. (comsonte)

trad. ang. : Sound in the momilh.

La confusion reside dans le fatt qu" un som yui se produit "dams la bouche". un son "huccal" pour ainsi dire, peut, au plan phonétique, etre sourd ou sonore. S'il est sonore, en raison de la resonance des cordes vociales, on voit difficilement comment ces périphratses alkans permetent de distinguer entre la voselle proprement dite tat lat consome sonore, etant donne que lat voix mervient dams les deux denominations ahans. In deuxiène exemple est relatif a la terminologic propose par Barlels et Annobil (1960): 1-2) dans leur grammaire fanti pour lat notion de melaphore.

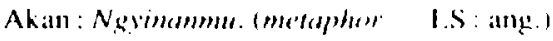

trad. frat. ('e qui remplace. Imétaphore)

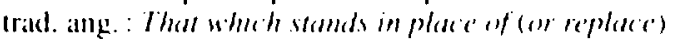

l.utilisation de cette periphrase. lexicalisede. entratine des chevauchements entre la métaphore el d'atures phénomene's lingussucues bases sur lat substitution. Il suftit, pour démontrer cette imprécision. d'evoguer une autre périphrase employéc par les mêmes atuteurs. "pronom"

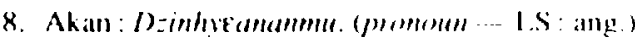

trad. frat : (ce qui remplace un nom. (promom)

trad. ang. : Thes which replare' a nom!

le pronom ćlant conçu comme lélentent qui remplace le nom (dzin), il est fort malaisé de déterminer la ligne de partage, car les deux appellations proposées par cette grammaire passent sous solence ke fait que la substitution metaphorigue, a la difference de la substitution pronominale, explote l'analogie pour operer un transfert de trats émantiques d'un référent à un alutre.

Pour terminer, nous aborderons brièvement d'attres méanismes qui, outre la translittération. la traduction et les formules paraphrastiques et périphrastiques. interviennent dans les langues africaines à des fins de désignation fechnique et semi-technique. Rappelons 
d'abord que certaines langues africaines comme l'akan sont dotées d'une faculté considérable, au niveau de la morphologie lexicale, de création de syntagmes nominaux qui en réalité sont des périphrases lexicalisées. Ce procédé, qui fait l'objet d'une large utilisation dans les grammaires akans citées plus haut, emploie des éléments morphologiques comme les suivants qui se retrouvent sous d'autres formes dans d'autres parlers africains:

(a) Akan : ... dze (ce qui permet de... ou sert à...) asembisadze (lexème ou élément interrogatlif) mpauparamudze (signe de ponctuation)

(b) Akan: ... bea (lieu, endroil)

Ce morphème locatif intervient dans la création de mots akans comme: as ndzibea (tribunal).

Dans les langues africaines, comme dans beaucoup d'autres langues, le registre semi-technique comporte certains mots qui au départ avaient une acception non technique mais qui par la suite ont revêtu un sens plus ou moins spécialisé. L'akan regorge de ce type de mots parmi lesquels figurent les lexèmes soulignés dans les exemples suivants tirés de Wonsom. Le vocable nsu (eau) prend un sens médical dans:

(a) Akan: W dze nsu sii no do. (He nas put on drip) trad. fra.: On a mis de l'eau sur lui. (On l'a mis sous perfusion) trad. ang.: They pat w'ater on him. texte: Wonsom (1986: 3).

(b) Akan : Dawurb 2 kratad. (new spaper) trad. fra. : Papier utilisé pour la diffusion de l'information. (journal)

Dans le deuxième exemple, le mot souligné dénote normalement la diffusion de messages et d'informations; dans le contexte africain. cela se fait assez. souvent à l'aide d'un instrument sonore appelé daw ur en akan. L'adjonction de krataa (papier) à da donc une expression ayant le sens plus ou moins technique de «journal», "revue». etc. II en est de même de certains éléments du vocabulaire métalinguistique employés par less grammairiens de l'akan. et qui sont issus de la langue de tous les jours. Entre autres, nyee et $d z i n$ qui signifient respectivement «action» et "nom» dans la lerminologie de la grammaire. Lés épithètes hunu et pa qui signifient communément "ordinaire, banal» et "véritable, réel» permettent, en grammaire, de démarquer le nom commun (din hunu) du nom propre (din pa) (Akrofi 1965).

Une troisième approche à signaler dans la constitution des registres plus ou moins spécialisés concerne la création d'appellations à partir des caractéristiques inhérentes ou fonctionnelles du référent dénommé. À cet égard, Namada (198:88) signale, à propos de la nomenclature botanique che\% les populations Nyindu du Zaïre, que les noms des espèces végétales en langue nyindu sont basés tantôt sur leurs propriétés fonctionnelles (leur destination). tantôt sur d'autres aspects comme la morphologie. I'habitat, etc. Par exemple, une plante grimpante ayant pour nom botanique agelaed de've'veri reçoit chez. les Nyindus le nom de kahukusingo (signification : partie supérieure et arrondie d'un panier), du fait de son utilisation dans le domaine de la vannerie. Cenains noms africains de maladies vétérinaires sont à rapprocher de ce procédé. Sidi Ba (1982: 8.3.86) note par exemple qu'une variole aviaire est appelée yokyok en peul car l'un des symptômes caractéristiques en est le son "yok yok yok" émis par les poulets lorsqu'ils en sont atteints.

Une autre procédure connexe est basée sur la dénomination par analogie explicite ou implicite. Andrzejewski (1971:266-268) indique que le lexeme technique pour «engin spatial" en somali, dayachgacmeed. signifie "lune fabriquée a la main» ou ahand-made moon». Cela suppose une analogie entre cet engin et la lune qui sont tous les deux suspendus dans l'espace. Le même auteur signale qu en somali, le verbe duub qui veut dire 
"enrouler (un fil. une corde)" a un sens technique ("enregistrer sur bande magnétique») qui laisse transparaitre une comparaison entre le mouvement de la bande magnétique lors de l'enregistrement du son el celui du fil qui senroule. Toujours en somali, le procédé analogique est sous-jacent aux suivants: afgembi (renverser un vaisseau) employé pour "coup d'Etat" et chidhiidhke siscasadea, terme technique pour "relations diplomatiques", dont le premier segment a trait a la corde servant at relier des chameaux dans une caravane (Andrejewski 1971). Il importe Également de noter que la plupart des langues africaines ont des lexèmes et des syntagmes propres pour un certain nombre de concepts qui sont techniques ou presque. On peut citer les exemples suivants en akan: oswka

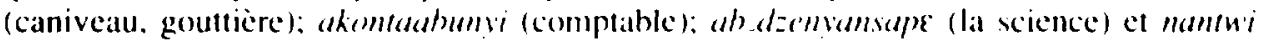
pomp (charbon -- terme vétérinatire).

Il ressort de ce qui précède que le français et l'anglais jouent un rôle linguistique important dans lexpression technique et semi-lechnique des langues africaines. Des exemples tirés d un certain nombre de langues africaines dont le peul, l'ewe, l'akan et le somali ont permis de démontrer que la traduction. la translittération et l'emploi des formules paraphrastiques et périphrastiques sont des procédes exploités par plusieurs langues africaines et qui supposent une activite de transfert linguistique partant du français ou de l'anglais comme langue source vers les lamgues africantes qui, de ce fait, deviennent des langues cibles. Il a été également démontre qüà côté de ces principaux outils linguisliques, la dynamique des langues africantes revide egalement dams l'existence, dans certains domaines, d'un lexique semi-technique el technique propre et aussi dans l'emploi d'autres possibilités de création lexicale è d'extension du sémantisme de mots déjà existants.

\section{REFERENCES}

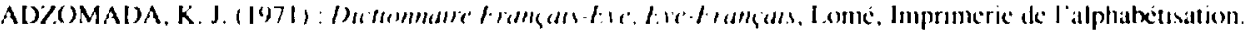

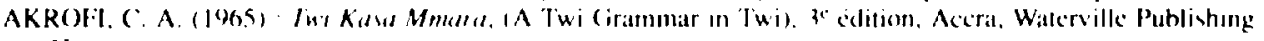
House.

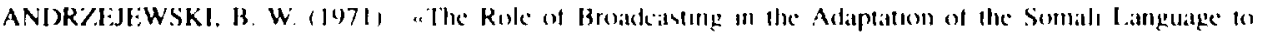

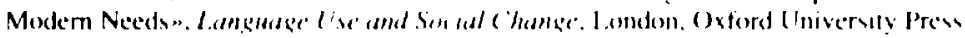

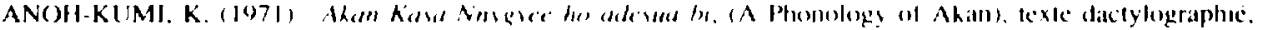
Universilé de (aipe ( isalse.

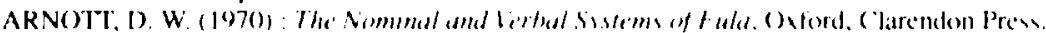

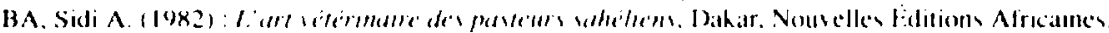

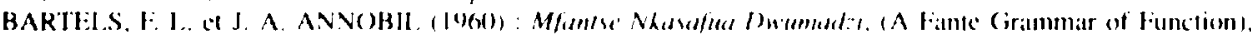

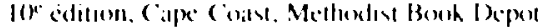

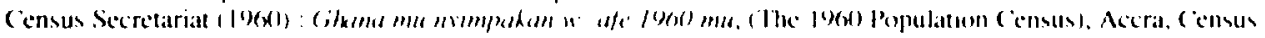
Secretarlat.

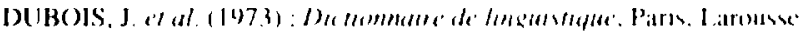

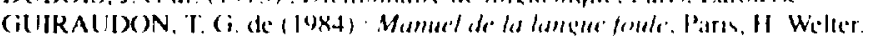

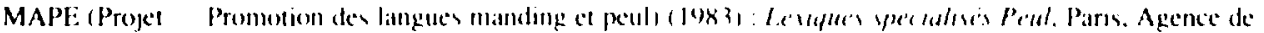
Cisoperation Culturelle et lestmicjuc.

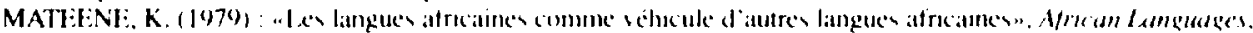
Mateene. Nuwachuhup. Dalby Editors, l.ondon, Internattonal Afruan Instetute

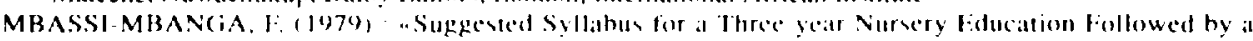

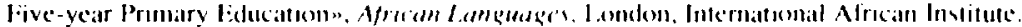

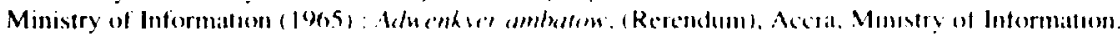

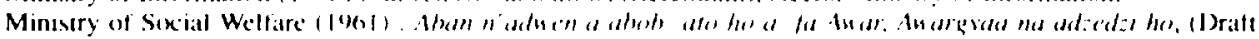

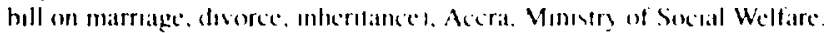

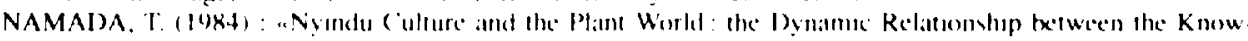

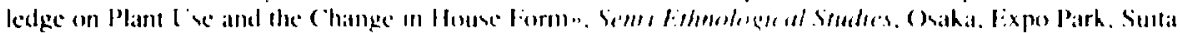

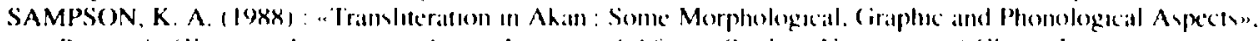

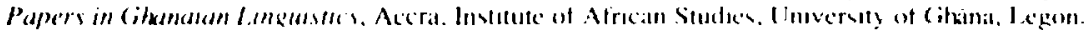

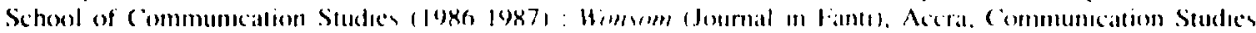
Prew. I niversily of (ihanit, leceon.

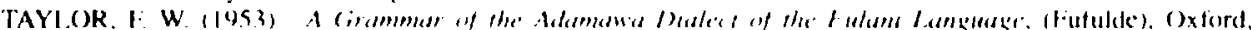
Clarendon Press. 